\title{
Efeitos de tarefas cognitivas no controle postural de idosos: Uma revisão sistemática
}

\author{
Effects of the cognitive tasks in the postural control of elderly: A \\ systematic revision
}

\author{
L.P. Andrade, F. Stella, F.A. Barbieri, N.M. Rinaldi, A.Y.Y. Hamanaka, L.T.B. Gobbi
}

\begin{abstract}
A demanda exigida de atenção para a manutenção do equilíbrio postural aumenta com o processo de envelhecimento, principalmente quando são realizadas duas ou mais tarefas concomitantes que exijam processamento mental. Trabalhos que têm abordado os efeitos de tarefas cognitivas no controle postural variam de acordo com o tipo da tarefa cognitiva executada e com o processo de envelhecimento, com possível interação destes dois componentes. O objetivo desta revisão foi identificar e analisar artigos publicados sobre os efeitos de tarefas cognitivas no controle postural de idosos. Para tanto, realizou-se uma busca sistemática nas seguintes bases de dados: Web of Science, SportDiscus, CINAHL, Science Direct on line, Biological Abstracts, PsycINFO e Medline. Foram encontrados 444 artigos, dos quais oito foram selecionados de acordo com os critérios de inclusão e exclusão previamente definidos. As publicações mostraram que o controle postural tende a ser influenciado por tarefas cognitivas do tipo atenção e que, em idosos, este fenômeno pode estar associado ao aumento no risco de quedas. Sugere-se que a maior tendência de ocorrer quedas em idosos é devido à execução simultânea de controle motor e processamento cognitivo que aumenta o grau de complexidade da tarefa.

Palavras-chave: tarefas cognitivas, controle postural, idosos, atenção, tarefa dupla
\end{abstract}

ABSTRACT

Demanding attention in order to keep postural balance increases with aging and with the presence of concurrent tasks that require information processing. Several studies have demonstrated that motor performance can be related to the complexity of the task and aging process, presenting a possible interaction between these factors. The aim of this review was to identify and analyze published papers about the effects of cognitive tasks on the postural control of elderly individuals. A systematic search in the Web of Science, SportDiscus, CINAHL, Science Direct on line, Biological Abstracts, PsycINFO, and Medline databases was made and 444 articles were found. Eight were selected that studied the variables of interest. These studies showed that postural control seems to be influenced by the individual's attention processes and that deficits in such ability may be associated to an increased risk of falls. Keywords: postural control, dual task, cognitive tasks, attention, aged

Submetido: 28.12.2009 | Aceite: 24.10.2010

Larissa Pires de Andrade, Fábio Augusto Barbieri, Natália Madalena Rinaldi, Ágata Yoko Yasue Hamanaka e Lilian Teresa Bucken Gobbi. Departamento de Educação Física, Laboratório de Atividade Física e Envelhecimento (LAFE) - Universidade Estadual Paulista (UNESP), Rio Claro, SP - Brasil.

Florindo Stella. Departamento de Educação, Laboratório de Atividade Física e Envelhecimento (LAFE) Universidade Estadual Paulista (UNESP), Rio Claro, SP - Brasil.

Endereço para correspondência: Lilian Teresa Bucken Gobbi, Departamento de Educação Física, Universidade Estadual Paulista (UNESP), Avenida 24-A, 1515 - Bela Vista, CEP: 13506-900 - Rio Claro/SP, Brasil.

E-mail: ltbgobbi@rc.unesp.br 
A capacidade de executar determinadas tarefas motoras diminui com o aumento da idade (Kenny, Yardley, Martineau, \& Jay, 2008). O idoso é capaz de manter o equilíbrio corporal quando está concentrado na realização de uma atividade específica, mas pode não apresentar o mesmo padrão de execução quando precisa realizar múltiplas tarefas simultaneamente (Jamet, Deviterne, Gauchard, Vançon, \& Perrin, 2006; Voelcker-Rehage \& Alberts, 2007). A atenção dividida ${ }^{1}$ na realização concomitante de várias atividades pode gerar dificuldades no desempenho motor e no equilíbrio (Jamet et al., 2006).

O controle postural é o processo de geração de padrões de atividades do sistema nervoso central para regular o relacionamento entre $o$ centro de massa e a base de suporte (Shumway-Cook \& Woollacott, 2003). O envelhecimento proporciona mudanças negativas no controle postural, causando perda da recepção sensorial, lentidão do processamento cognitivo e dificuldade na execução da resposta motora exigida (Maki \& Mcllroy, 1996; Duncan, Chandler, Studenski, Hughes, \& Prescott, 1993). Os déficits nestas três funções do processamento e execução das tarefas são causados por alterações em diferentes estruturas cerebrais, tais como núcleo vestibular (Lopez, Honrubia, \& Baloh, 1997), cerebelo (Rauch, Velásquez-Villasenõr, Dimitri, \& Merchant, 2001) e redução do tecido cerebral que se observa pelo alargamento dos ventrículos cerebrais (Furman \& Redfern, 2001), podendo agravar a deficiência do equilíbrio. A redução da massa muscular e da força (Mackey \& Robinovitch, 2006; Tell, Lefkowitz, Diehr, \& Elster, 1998), decorrentes do envelhecimento, também podem reduzir consideravelmente o padrão de execução motora.

Particularmente, as alterações nos sistemas sensoriais e musculoesquelético afetam de alguma forma o controle postural (Judge,

\footnotetext{
1 Atenção dividida: Durante o processo atencional, alternância do foco em mais de um estímulo relevante ao mesmo tempo (Schmidt \& Wrisberg, 2001).
}

2003). Déficits nestes sistemas podem interferir no controle antecipatório e reativo. Os componentes sensoriais e musculoesqueléticos contribuem para o controle da posição relativa dos segmentos corporais entre si e com o ambiente, além do controle das forças que agem sobre o corpo (Horak \& Macpherson, 1996). Para tentar integrar estes sistemas e manter a postura ereta, os idosos apresentam maior deslocamento e variabilidade do centro de pressão na direção ântero-posterior (Amiridis, Hatzitaki, \& Arabatzi, 2003; Van Wegen, Emmerik, \& Riccio, 2002). Entretanto, nas posições com o pé dominante à frente e unipodal há maior deslocamento do centro de pressão na direção médio-lateral como forma de se evitar a queda (Amiridis et al., 2003; Van Wegen et al., 2002).

A manutenção da postura ereta de idosos pode ser prejudicada quando mais de uma tarefa é realizada, necessitando-se de ajustes apropriados e da integração dos componentes do controle postural para evitar o fenômeno de queda. Quando uma tarefa cognitiva é executada na postura ereta, a atenção tende a ser dividida entre o controle postural e o processamento cognitivo (Woollacott \& Shumway-Cook, 2002). A execução de uma tarefa motora com demanda cognitiva relativamente baixa pode beneficiar o controle postural, orientando o indivíduo a manter a atenção neste tipo de controle, enquanto que uma demanda cognitiva elevada tende a prejudicar a regulação da oscilação postural (Huxhold, Li, Schmiedek, \& Lindenberg, 2006). No entanto, há uma lacuna no entendimento do relacionamento entre os processos e os componentes envolvidos na manutenção da postura aliada à execução de uma tarefa cognitiva, sendo importante a realização de estudos que abordem os aspectos motores e cognitivos relacionados ao controle postural desta população (Fraizzer \& Mittra, 2007).

Neste contexto, o objetivo deste estudo é, por meio de uma revisão sistemática, 
identificar e analisar a associação entre tarefas cognitivas e controle postural em idosos. A revisão sistemática permite sintetizar informações disponíveis sobre um determinado assunto, auxiliando clínicos e pesquisadores no seu cotidiano de trabalho (Sampaio \& Mancini, 2007). O entendimento da influência de tarefas cognitivas no controle postural de idosos pode favorecer a redução de quedas.

\section{MÉTODO}

Para se atingir o objetivo proposto, uma revisão com busca sistemática das publicações sobre a influência de tarefas cognitivas no controle postural de idosos foi realizada. A busca incluiu as seguintes bases de dados: Web of Science, SportDiscus, CINAHL, Science Direct on line, Biological Abstracts, PsycINFO e Medline. O período considerado para a inclusão das publicações abrangeu todos os anos de edição dos periódicos até março de 2008. Para a estratégia de identificação dos estudos, consideraram-se as seguintes palavras-chave e operadores boleanos: (Posture control OR Static Posture OR Posture) AND (Dual task ${ }^{2}$ OR Cognitive task OR Attention ${ }^{3}$ ) AND (Elderly OR Older Adults OR Aged).

A seleção dos artigos baseou-se nos seguintes critérios de inclusão: estudos controlados e randomizados que envolvessem, concomitantemente, tarefas motoras que exigissem controle postural e tarefas cognitivas; estudos com sujeitos com idade a partir de 65 anos; estudos que utilizassem plataforma de força para se mensurar a oscilação do centro de pressão. Os critérios de exclusão adotados foram: ausência de análise da interação do controle postural

\footnotetext{
${ }^{2}$ Tarefa dupla: técnica de pesquisa para "determinar as características e as solicitações da atenção em duas tarefas diferentes desempenhadas simultaneamente" (Magill, 2000, p. 113).

${ }^{3}$ Atenção: refere-se à seleção de um estímulo sensorial particular ou de um processo mental para análise posterior (Purves et al., 2004). No contexto do desempenho humano, atenção envolve processos perceptivos, cognitivos e motores associados ao desempenho de habilidades (Magill, 2000).
}

simultaneamente com a execução de tarefas cognitivas; estudos de revisão da literatura.

De acordo com as palavras-chave e operadores boleanos foram identificados 444 artigos. Primeiramente, todos os artigos foram avaliados segundo informações oriundas do título, resumo e palavras-chave. Posteriormente, analisaram-se na íntegra os artigos que atendiam parcialmente aos critérios de inclusão, examinando-se os seguintes itens desses artigos: a) objetivos; b) sujeitos; c) tarefas cognitivas; d) procedimentos para coleta de dados tarefas motoras; e) resultados obtidos. A maioria dos estudos comparou a influência da cognição no controle postural entre jovens e idosos. Finalmente, com base nesta análise, oito artigos (Tabela 1) preenchiam integralmente os critérios de inclusão estabelecidos.

\section{RESULTADOS}

Estudo 1 (Teasdale, Stelmach, Bard, \& Fleury, 1992): Neste estudo, os autores avaliaram, comparativamente, em idosos e jovens a interferência da manutenção da atenção em um determinado foco visual, com alternância da condição de olhos abertos e de olhos fechados, na manutenção da postura em uma plataforma de força. As oscilações corporais, nas direções antero-posterior e médio-lateral foram registradas pela plataforma de força. Os participantes permaneceram em pé, descalços, com os pés juntos, e com o olhar dirigido a três metros em um ponto fixo. Cinco tentativas de 180 segundos foram coletadas. Para cada tentativa, estímulos auditivos eram liberados, a fim de estimular os sujeitos a abrir e fechar os olhos. Para análise dos dados, valores de referências, calculados a partir das oscilações corporais avaliadas durante $\mathrm{o}$ experimento, foram utilizados. As oscilações corporais obtidas, depois das transições sem visão e com visão, foram comparadas com os valores de referências para determinar se os idosos poderiam reintegrar rapidamente a adição do sistema sensorial visual, vestibular e proprioceptivo. Para ambos os grupos, os 
valores obtidos das oscilações corporais, depois das transições, foram sempre maiores que os de referência. Resultados similares foram obtidos para a variabilidade do centro de pressão. Em comparação com os jovens, os idosos apresentaram aumento da oscilação corporal. Na transição com visão, os jovens estabilizaram a postura mais rapidamente, do que quando comparados com os idosos. Além disso, os idosos apresentaram maior dispersão do equilíbrio, após a transição sem visão/visão e depois da transição visão/sem visão.

Estudo 2 (Teasdale, Bard, Larue, \& Fleury, 1993): O segundo estudo encontrado é similar ao primeiro. O objetivo foi investigar se a demanda de atenção, para manter a postura, aumenta com a idade e varia com a qualidade das informações sensoriais disponíveis. A tarefa cognitiva realizada, neste estudo, também foi de atenção, mensuradas por meio do tempo de reação, onde os sujeitos eram orientados a apertar, tão rápido quanto possível, um botão ao ouvirem um estímulo auditivo. Para isso, duas avaliações (sentada e em pé) foram realizadas previamente para obter valores controles do tempo de reação. Logo depois, o participante era convidado a ficar em pé novamente na plataforma de força com os pés juntos e braços ao longo do corpo, com o olhar dirigido a um alvo a três metros de distância. Os estímulos auditivos foram apresentados de forma aleatória. Na condição em que eles permaneciam em pé na plataforma de força, quatro situações experimentais foram empregadas: i) olhos abertos e superfície normal; ii) olhos fechados e superfície normal; iii) olhos abertos e superfície alterada (espuma de poliuretano $5 \mathrm{~cm}$ de espessura na plataforma de força); e iv) olhos fechados e superfície alterada. Em comparação com os jovens, os idosos apresentaram um grau maior de dificuldade de manutenção do equilíbrio postural nas quatro condições, apresentando maior deslocamento do centro de pressão. Com a diminuição da informação sensorial, a tarefa postural tornou-se cada vez mais difícil para os idosos e exigiu mais da sua capacidade de atenção, com aumento do tempo de reação ${ }^{4}$.

Estudo 3 (Marsh \& Geel, 2000): Também neste estudo, os autores compararam a manutenção do equilíbrio postural em idosas e jovens durante a exigência de atenção visual e auditiva, em uma plataforma de força. O estímulo auditivo foi aplicado para se aferir o tempo de reação. Duas tarefas foram empregadas: participantes sentados (tarefa primária) e em pé (tarefa secundária). $\mathrm{Na}$ condição em pé e descalços, quatro condições experimentais combinando os fatores visão (olhos abertos e fechados) e superfície (rígida e com espuma) foram solicitadas. Houve aumento da oscilação corporal nas condições com os olhos fechados e nas com a superfície com espuma, em ambos os grupos. As idosas apresentaram maior deslocamento, velocidade e área do centro de pressão quando se exigia informação visual, bem como, apresentaram um maior tempo de reação ao estímulo auditivo.

Estudo 4 (Teasdale \& Simoneau, 2001): Neste estudo, idosos foram comparados com adultos quanto à manutenção do equilíbrio postural na presença de condições variáveis quanto à visão (olhos abertos e fechados) e proprioceptivos (superfície regular e irregular), em uma plataforma de força. O objetivo do estudo foi examinar se a reintegração das informações sensoriais prejudica o equilíbrio e requer mais demanda atencional. Como tarefa dupla, os participantes responderam em voz alta, o mais rápido possível, a um estímulo auditivo imprevisível, apresentado antes ou após a reintegração sensorial e em condições de controle (manutenção do equilíbrio corporal). O tempo de reação aos estímulos auditivos foi utilizado para determinar as demandas de atenção para o sistema do controle postural. A reintegração de propriocepção em ambas as condições de visão aumentou a velocidade do centro de pressão

\footnotetext{
${ }^{4}$ Tempo de reação: Intervalo entre a apresentação de um estímulo e o início da resposta (Schmidt \& Wrisberg, 2001).
} 
para todos os participantes. Este efeito, porém, era maior para os idosos do que para os jovens. Um aumento da demanda de atenção foi observado para ambos os grupos, principalmente quando as informações proprioceptivas foram alteradas. Os idosos foram mais lentos para responder ao estímulo auditivo, à reintegração da propriocepção nas condições visão sem visão.

Estudo 5 (Brauer, Woollacott, \& ShumwayCook, 2002): Os autores investigaram a interferência de uma tarefa cognitiva, com processamento atencional auditivo e resposta verbal, com medida do tempo de reação, na manutenção do equilíbrio postural em uma plataforma de força. Na plataforma de força foram realizadas perturbações para a avaliação da manutenção na instabilidade postural. Para tanto, jovens e idosos que não sofriam quedas rotineiras (não caidores) e idosos que sofriam quedas rotineiras (caidores) foram avaliados. Os resultados mostraram tempo de reação maior para todo os participantes na tarefa dupla. Ao realizar a tarefa cognitiva concomitante com uma tarefa de recuperação de equilíbrio, os idosos priorizaram a recuperação de equilíbrio com um passo. Os idosos caidores utilizaram um passo ainda maior para recuperar o equilíbrio do que quando comparados aos outros dois grupos (idosos não caidores e jovens). Já os jovens conseguiram realizar as tarefas simultaneamente. Entretanto, essas diferenças não foram significativas entre o centro de pressão nos três grupos.

Estudo 6 (Jamet et al., 2006): Os autores investigaram, comparativamente, idosos, adultos e jovens quanto à manutenção do equilíbrio postural em uma plataforma de força em três condições: i) tarefa mental - cálculo regressivo de sete em sete; ii) tarefa visuoverbal com a ajuda do Stroop Color Test; e iii) tarefa audioverbal. Na primeira condição, os idosos apresentaram maior deslocamento e área do centro de pressão quando comparados com os adultos e os jovens. $\mathrm{Na}$ segunda condição, houve melhora no controle postural nos jovens e nos adultos, mas não nos idosos. $\mathrm{Na}$ terceira condição, não houve aumento significativo de deslocamento e de área do centro de pressão nos três grupos. Entretanto, é importante ressaltar que, nessa condição, os jovens apresentaram uma leve tendência de melhora do controle postural. Houve correlação positiva entre o grau de dependência visual e a tarefa cognitiva.

Estudo 7 (Huxhold et al., 2006): Este trabalho comparou idosos e adultos quanto à manutenção do equilíbrio postural, em uma plataforma de força, com e sem tarefa cognitiva que exigia atenção e verbalização. Três tarefas cognitivas diferentes foram realizadas na plataforma de força. Em todas as tarefas os participantes permaneciam em pé, segurando um botão em cada mão, e com os braços ao longo do corpo. $\mathrm{Na}$ tarefa simples, os participantes permaneceram com o olhar dirigido a um alvo. $\mathrm{Na}$ tarefa dupla os participantes deveriam apenas permanecer em pé, assistindo uma série de 22 números aleatórios, permanecendo o mais estável possível. As tarefas cognitivas foram a de tempo de reação, memória de trabalho e visuoespacial. A tarefa de tempo de reação consistia em mostrar 22 números, entre um e nove na tela de um computador. Os números 1 e 3 estavam destacados. À medida que os números eram apresentados aleatoriamente aos participantes, eles deveriam apertar o botão com uma das mãos. Na tarefa de memória de trabalho, os participantes deveriam lembrar e relatar os dois números anteriores aos números apresentados com marcações. $\mathrm{Na}$ tarefa visuo-espacial, um ponto era inserido em alguma parte de uma tabela de linhas e colunas com oito quadros. Vinte e dois pontos foram inseridos aleatoriamente, no quadro, em diferentes localizações. Quando aparecia um ponto marcado, o participante deveria lembrar os dois pontos imediatamente anteriores. Quando os participantes realizaram tarefas cognitivas fáceis, a demanda de atenção exigida se voltava para o controle postural, de modo que o deslocamento do centro de pressão 
diminuía. Entretanto, nas tarefas de maior demanda cognitiva, os idosos apresentaram maior deslocamento do centro de pressão. Em relação à resposta ao tempo de reação, os idosos erraram mais, e foram mais lentos quando comparados com os jovens.

Estudo 8 (Doumas, Smolders, \& Krampe, 2008): Nesta investigação, foram comparados, idosos e adultos quanto à manutenção do equilíbrio postural, em uma plataforma de força em duas sessões. Na primeira sessão, quatro condições foram realizadas: i) postura estável; ii) com informação visual; iii) com informação somatossensorial; e iv) tarefa de memória de trabalho de contagem regressiva. $\mathrm{Na}$ segunda sessão, as condições 1 e 4 foram repetidas. Os participantes apresentaram maior deslocamento do centro de pressão, nas condições com informações visuais e somatossensoriais. No desempenho da tarefa dupla, os idosos apresentaram quase $40 \%$ de aumento da instabilidade postural, em comparação com a tarefa simples.

\section{DISCUSSÃO}

Os estudos demonstraram que a manutenção da postura ereta concomitante com tarefas que requerem atenção pode influenciar as oscilações corporais em idosos. Consequentemente, a oscilação postural foi aumentada, sendo que o comprometimento cognitivo, pode estar associado com o envelhecimento e considerado fator importante para o aumento da oscilação no controle postural (Häkkinen, Pastinen, Karsikas, \& Linnamo, 1995).

Aliado a isso, idosos apresentam dificuldades em captar a informação visual para controlar as oscilações corporais (Paulus, Straube, Krafczyk, \& Brandt, 1989; Teasdale et al., 1992). Os idosos são mais instáveis que adultos jovens nas condições que alteram as informações sensoriais, sendo que a diferença entre as idades só aparece quando as informações sensoriais estão comprometidas (Teasdale et al., 1992).
O processamento sensorial parece ser sensível ao declínio da idade, o que pode ser verificado pela instabilidade postural. Quando a superfície de apoio foi manipulada para realização da tarefa dupla, os idosos apresentaram aumento em torno de $40 \%$ na instabilidade postural para tentar realizar a tarefa cognitiva com sucesso (Doumas et al., 2008). Entretanto, quando a superfície de suporte foi alterada (informação somatossensorial referenciada), a estabilidade postural não foi modificada durante a realização de tarefa simples e dupla (Doumas et al., 2008). Estes resultados sugerem que idosos são menos flexíveis cognitivamente, pois não conseguiram controlar as instabilidades posturais. Isto é sugerido nas experiências de exigência de atenção juntamente com a manutenção do equilíbrio descritos nos artigos analisados.

Aliado às mudanças na instabilidade postural, idosos apresentam aumento no tempo de reação quando expostos a estímulos visuais e auditivos se comparados a jovens na realização de tarefas duplas. Este aumento no tempo de reação acontece principalmente em tarefas cognitivas que demandam atenção e memória, referência visual e somatossensorial (Tell et al., 1998; Häkkinen et al., 1995; Judge, 2003; Amiridis et al., 2003), indicando maior dificuldade e estratégias diferenciadas dos idosos em relação a adultos jovens (Doumas et al., 2008). Os idosos priorizam a manutenção do equilíbrio recuperado, pois não conseguem realizar a tarefa de manutenção postural concomitante com tarefas cognitivas, enquanto que os adultos jovens realizam estas tarefas simultaneamente (Brauer et al., 2002). É provável que, devido ao processo de envelhecimento, déficits na integração sensorial de idosos ocorrem, o que prejudica seus desempenhos nas tarefas duplas. $O$ aumento da idade reduz a capacidade no processamento cognitivo, com prejuízo para a habilidade de dividir a atenção e transferir apropriadamente os recursos entre tarefas concorrentes (Brauer et al., 2002; Chevignard, 
Tabela 1

Descrição sucinta dos estudos que examinaram os efeitos da tarefa cognitiva (TC) no controle postural (CP) de idosos

\begin{tabular}{|c|c|c|c|c|}
\hline Estudo & Participantes & Objetivo & Tarefas & Resultados \\
\hline $\begin{array}{l}\text { 1. Teasdale et } \\
\text { al. (1992) }\end{array}$ & $\begin{array}{c}7 \text { idosos } \\
\text { (72.4 anos) } \\
7 \text { jovens } \\
\text { (21.6 anos) }\end{array}$ & $\begin{array}{l}\text { Manutenção da } \\
\text { atenção no foco } \\
\text { visual }\end{array}$ & $\begin{array}{l}\text { Manter a postura: } \\
\text { olhos abertos e/ou } \\
\text { fechados, além de } \\
\text { atenção no foco visual }\end{array}$ & $\begin{array}{l}\text { Idosos: maior oscilação } \\
\text { do CP com alteração } \\
\text { entre condição de olhos } \\
\text { abertos e olhos fechados. }\end{array}$ \\
\hline $\begin{array}{l}\text { 2. Teasdale et } \\
\text { al. (1993) }\end{array}$ & $\begin{array}{l}9 \text { idosos } \\
\text { (71.1 anos) } \\
8 \text { adultos } \\
\text { (24.6 anos) }\end{array}$ & $\begin{array}{l}\text { Demanda atencional } \\
\text { no CP relacionado } \\
\text { com a idade e } \\
\text { relacionado com a } \\
\text { qualidade da } \\
\text { informação sensorial }\end{array}$ & $\begin{array}{c}\text { Manter a postura: } \\
\text { olhos abertos e/ou } \\
\text { fechados superfícies } \\
\text { normais e/ou } \\
\text { alteradas, além de } \\
\text { atenção aos estímulos } \\
\text { auditivos }\end{array}$ & $\begin{array}{l}\text { Idosos: menor } \\
\text { informação sensorial, } \\
\text { maior dificuldade } \\
\text { postural e maior } \\
\text { requerimento atencional. }\end{array}$ \\
\hline $\begin{array}{l}\text { 3. Marsh e } \\
\text { Geel (2000) }\end{array}$ & $\begin{array}{c}16 \text { idosos } \\
(71.5 \pm 3.4 \text { anos }) \\
14 \text { adultos } \\
(25.5 \pm 2.4 \text { anos })\end{array}$ & $\begin{array}{l}\text { Demanda atencional } \\
\text { no controle postural }\end{array}$ & $\begin{array}{l}\text { Manter postura: olhos } \\
\text { abertos e fechados } \\
\text { sem estímulo auditivo } \\
\text { e com estímulo } \\
\text { auditivo, superfície } \\
\text { rígida e com espuma }\end{array}$ & $\begin{array}{c}\text { Idosos: maior oscilação } \\
\text { do CP associado à maior } \\
\text { demanda atencional e } \\
\text { maior TR. }\end{array}$ \\
\hline $\begin{array}{l}\text { 4. Teasdale e } \\
\text { Simoneau } \\
(2001)\end{array}$ & $\begin{array}{c}8 \text { idosos } \\
(68.0 \text { anos }) \\
8 \text { adultos } \\
(24.8 \text { anos })\end{array}$ & $\begin{array}{l}\text { Influência da idade } \\
\text { no CP e requisição } \\
\text { de maior demanda } \\
\text { atencional }\end{array}$ & $\begin{array}{l}\text { Manter postura em } \\
\text { condições variáveis de } \\
\text { tarefas com e sem } \\
\text { visão e propriocepção, } \\
\text { verificando TR em } \\
\text { comportamento verbal } \\
\text { a estímulo auditivo }\end{array}$ & $\begin{array}{l}\text { Idosos: maior oscilação } \\
\text { do CP na tarefa de maior } \\
\text { demanda de atenção e } \\
\text { perturbação do CP. }\end{array}$ \\
\hline
\end{tabular}

15 idosos - não caidores

(72.1 \pm 7.0 anos)

5. Brauer et al. 13 idosos (2002) caidores Efeito TC na (79.2 \pm 7.0 anos) resposta de atenção 15 jovens (23.0 \pm 5.0 anos) 28 idosos (71.0 \pm 7.0 anos)

6. Jamet et al. 26 jovens (2006) (22.0 \pm 2.0 anos) 26 adultos (43.0 \pm 8.0 anos)
Efeito da idade na mobilização cognitiva e CP
Manter a postura: mediante estímulo auditivo com resposta verbal e perturbação da PF
Idosos: apresentaram quando demanda atencional foi exigida. tempo de reação maior

$$
19 \text { idosos }
$$

7. Huxhold et $(69.8 \pm 3.3$ anos $)$ Relação entre CP e al. (2006) TC

$$
20 \text { adultos }
$$

$(24.5 \pm 2.6$ anos $)$

\begin{tabular}{cc}
\hline 8. Doumas, & 18 idosos \\
Smolders e & $(70.9 \pm 3.4$ anos $)$ \\
Krampe (2008) & 18 adultos \\
& $(21.7 \pm 2.1$ anos $)$
\end{tabular}

\section{Mudanças no CP de} acordo com a informação sensorial realizada concomitantemente
Manter a postura: tarefa simples e com TC mental, visuoverbal e visuoauditivo
Idosos: maior oscilação AP e ML do CP em tarefa concomitante.
Manter a postura: Idosos: maior tarefa simples; tarefa dupla assistindo números; três tarefas cognitivas verbais

em relação aos adultos e menor deslocamento do COP em tarefa dupla. Manter a postura: com instabilidade postural TC que demandam atenção e memória quando submetidos a TC com demanda de atenção e memória.

Nota: TR: tempo de reação; AP: oscilação ântero-posterior; ML: oscilação médio-lateral; PF: plataforma de força 
Taillefer, Poncet, Picq, \& Pradat-Diehl, 2008; Häkkinen et al., 1995).

Os processos cognitivos, especialmente aqueles relacionados à atenção, influenciam decisivamente no controle postural de idosos (Jamet et al., 2006). Os oito artigos que compõem esta revisão indicaram este fato, especialmente quando jovens adultos e idosos foram comparados. Nas situações em que o indivíduo divide sua atenção na execução de uma atividade cognitiva concomitante com tarefas de manutenção postural, parte dos recursos atencionais é deslocada para esta atividade cognitiva (Huxhold et al., 2006). Com isso, o controle postural pode ficar comprometido e tende a ocorrer aumento no número de quedas. Isto significa que o comprometimento do controle postural de idosos está relacionado com o grau de exigência da tarefa cognitiva (Jamet et al., 2006). Quanto mais exigente é a tarefa cognitiva, maior é o aumento da oscilação do centro de pressão, porém quando a atividade cognitiva com menor grau de exigência atencional é realizada concomitantemente com tarefas de manutenção da postura ereta, atenuações na oscilação corporal podem ser observadas (Woollacott \& Shumway-Cook, 2002).

Outro componente importante para o controle postural é a visão. Vários estudos analisados evidenciaram que a manipulação da informação visual interfere no desempenho dos idosos na tarefa de manutenção postural (Doumas et al., 2008; Jamet et al., 2006; Marsh \& Geel, 2000; Teasdale et al., 1992; Teasdale \& Simoneau, 2001;). Como resultado, os idosos aumentaram a oscilação do centro de pressão quando realizaram a tarefa dupla de manutenção postural sem visão e acionar um botão em função de um estímulo sonoro. Uma possível explicação para este resultado é a reduzida capacidade de processamento de informação que compromete a habilidade de se dividir a atenção entre duas tarefas. Ainda, a reduzida sensibilidade periférica aumenta $\mathrm{o}$ grau de complexidade exigida pelos mecanismos centrais de controle e, consequen- temente, esta sensibilidade alterada afeta desfavoravelmente os recursos atencionais. Em função disto, o desempenho da tarefa postural requer maiores níveis de atenção (Teasdale et al., 1992).

Além das tarefas apresentadas anteriormente, também foi investigada a inserção de outras funções cognitivas no estudo da associação entre demanda de atenção e controle postural. A investigação de Woollacott e Shumway-Cook (2002) e dois estudos incluídos nesta revisão, coordenados por Jamet et al. (2006) e por Doumas et al. (2008) evidenciaram esta associação. Nesta linha de raciocínio, estes estudos introduziram tarefas de memória ou cálculo, juntamente com demanda de atenção, na execução do controle postural. Também foi verificado que a oscilação do centro de pressão era maior quando as tarefas posturais exigiam processamento cognitivo com maior grau de complexidade, como mostra o estudo de Huxhold et al. (2006). Por exemplo, a manipulação da posição, em pé e sentado, e também da visão, olhos abertos e fechados, pode aumentar a necessidade de recursos cognitivos de idosos para controlar a postura. Desta forma, é importante inferir que a combinação de restrições quanto à posição e à informação visual pode aumentar o risco de quedas para idosos quando realiza duas tarefas concomitantes.

Juntamente com a manipulação destes aspectos, o peso sensorial atribuído à informação somatossensorial pode ser maior para os idosos do que para os jovens (Teasdale \& Simoneau, 2001). Na ausência de visão, as informações somatossensorial e vestibular devem ser reintegradas, com demanda adicional de atenção em ambos os grupos. Em geral, mudanças no processamento das informações sensoriais reduzem a capacidade de regulação pelos mecanismos centrais responsáveis pela organização do controle postural de idosos, aumentando o risco de perda do equilíbrio (Teasdale \& Simoneau, 2001). Com o envelhecimento, há redução na 
capacidade de alocação dos recursos atencionais para a realização concomitante de tarefas diferentes (Teasdale et al., 1992, 1993). Ainda, a diminuição da disponibilidade de informação sensorial periférica também exige maior demanda de atenção em procedimentos que requeiram controle postural (Teasdale et al., 1992). Os achados da presente análise reforçam o conceito de que o controle postural é um fenômeno complexo e que exige a interdependência de sistemas sensoriais e motores periféricos e de ações cognitivas processadas em nível superior de controle (Teasdale et al., 1992, 1993; Teasdale \& Simoneau, 2001). Neste paradigma de tarefas duplas, os recursos de atenção alocados para a realização de tarefas cognitivas podem influenciar o desempenho do controle postural (Teasdale et al., 1993; Teasdale \& Simoneau, 2001).

\section{CONCLUSÕES}

Os estudos analisados confirmam a existência de relação entre o controle postural e desempenho em tarefas cognitivas. Tarefas cognitivas, especialmente as que demandam atenção, exercem influência no controle postural quando estes dois tipos de fenômenos são executados concomitantemente. Esta influência pode ser constatada pelo aumento do tempo de reação a um estímulo visual ou auditivo associado à manutenção da postura ereta. Neste procedimento, a inserção de tarefas cognitivas que exigem atenção relacionou-se com as tarefas de controle postural. Quando a manutenção da postura era efetuada concomitantemente com desempenho em tarefas que exigiam atenção, cálculo e memória de trabalho, observou-se aumento relevante da oscilação corporal nos idosos estudados. Portanto, o aumento na oscilação corporal durante a manutenção da postura em pé, quando realizada concomitantemente com tarefas cognitivas que exijam o processamento principalmente da atenção, pode representar aumento relevante do risco de quedas em idosos.

\section{REFERÊNCIAS}

Amiridis, I. G., Hatzitaki, V., \& Arabatzi, F. (2003). Age-induced modifications of static postural control in humans. Neuroscience Letters, 350, 137-140.

Brauer, S. G., Woollacott, M., \& Shumway-Cook, A. (2002). The influence of a concurrent cognitive task on the compensatory stepping response to a perturbation in balance-impaired and healthy elders. Gait \& Posture, 15(1), 83-93.

Chevignard, M., Taillefer, C., Poncet, F., Picq, C., \& Pradat-Diehl, P. (2008). The effect of age on executive functioning after acquired brain injury in adults. Revista de Neurología, 164(12), 1018-1027.

Doumas, M., Smolders, C., \& Krampe, R. T. (2008). Task prioritization in aging: Effects of sensory information on concurrent posture and memory performance. Experimental Brain Research, 187, 275-281.

Duncan, P., Chandler, J., Studenski, S., Hughes, M., \& Prescott, B. (1993). How do physiological components of balance affect mobility in elderly men? Archives of Physical Medicine and Rehabilitation, 74, 1343-1349.

Fraizzer, E. V., \& Mittra, S. (2007). Methodological and interpretive issues in posture-cognition dual-tasking in upright stance. Gait $\&$ Posture, 27, 271-279.

Furman, J. M., \& Redfern, M. S. (2001). Effect of aging on the otolith-ocular reflex. Journal of Vestibular Research, 11, 91-103.

Häkkinen, K., Pastinen, U.-M., Karsikas, R., \& Linnamo, V. (1995). Neuromuscular performance in voluntary bilateral and unilateral contraction and during electrical stimulation in men at different ages. European Journal of Applied Physiology, 70, 518-527.

Horak, F. B., \& Macpherson, J. M. (1996). Postural orientation and equilibrium. In L. B. Rowell \& J. T. Shepherd (Eds.), Handbook of physiology: A critical, comprehensive preservation of physiological knowledge and concepts (pp. 255-292). New York: Oxford.

Huxhold, O., Li, S. C., Schmiedek, F., \& Lindenberg, U. (2006). Dual-tasking postural control: Aging and the effects of cognitive demand in conjunction with focus of attention. Brain Research Bulletin, 69(3), 294-305.

Jamet, M., Deviterne, D., Gauchard, G., Vançon, G., \& Perrin, P. P. (2006). Age-related part taken by attentional cognitive processes in standing 
postural control in a dual-task context. Gait $\mathcal{E}$ Posture, 25(2), 179-184.

Judge, J. O. (2003). Balance training to maintain mobility and prevent disability. American Journal of Preventive Medicine, 25(3), 150-156.

Kenny, G. P., Yardley, J. E., Martineau, L., \& Jay, O. (2008). Physical work capacity in older adults: Implications for the aging worker. American Journal of Industrial Medicine, 51, 610-625.

Lopez, I., Honrubia, V., \& Baloh, R. W. (1997). Aging and the human vestibular nucleus. Journal Vestibular Research, 7, 77-85.

Mackey, D. C., \& Robinovitch, S. N. (2006). Mechanisms underlying age-related differences in ability to recover balance with the ankle strategy. Gait \& Posture, 23, 59-68.

Magill, R. A. (2000). Aprendizagem motora: Conceitos e aplicações ( $5^{\mathrm{a}}$ ed.). São Paulo, SP: Editora Edgard Blücher.

Maki, B. E., \& Mcllroy, W. E. (1996). Postural control in the older adult. Clinics in Geriatric Medicine, 12, 635-658.

Marsh, A. P., \& Geel, S. E. (2000). The effect of age on the attentional demands of postural control. Gait \& Posture, 12(2), 105-113.

Paulus, A., Straube, S., Krafczyk, \& Brandt, T. (1989). Differential effects of retinal target displacement, changing size and changing disparity in the control of anterior/posterior and lateral body sway. Experimental Brain Research, 78, 243-252.

Purves, D., Augustine, G. J., Fitzpatrick, D., Hall, W. C., LaMantia, A.-S., McNamara, J. O., \& Williams, S. M. (2004). Neuroscience ( $3^{\text {rd }}$ ed.). Massachusetts: Sinauer Publishers.

Rauch, S. D., Velásquez-Villasenõr, L., Dimitri, P. S., \& Merchant, S. N. (2001). Decreasing hair cell counts in aging humans. Annals of the New York Academy of Sciences, 942, 220-227.

Sampaio, R. F., \& Mancini, M. C. (2007). Estudos de revisão sistemática: Um guia para síntese criteriosa da evidência científica. Revista Brasileira de Fisioterapia, 11, 83-89.
Schmidt, R. A., \& Wrisberg, C. A. (2001). Processando informações e tomando decisões. In R. A. Schmidt, \& C. A. Wrisberg (Eds.), Aprendizagem e performance motora: Uma abordagem da aprendizagem baseada no problema (2 ed., pp. 69-101). Porto Alegre, RS: Artmed Editora.

Shumway-Cook, A., \& Woollacott, M. H. (2003). Controle postural. In A. Shumway-Cook, \& M. H. Woollacott (Eds.), Controle motor: Teoria e aplicações práticas (pp. 2-78). São Paulo: Manole.

Teasdale, N., \& Simoneau, M. (2001). Attentional demands for postural control: The effects of aging and sensory reintegration. Gait \& Posture, 14(3), 203-210.

Teasdale, N., Bard, C., Larue, J., \& Fleury, M. (1993). On the cognitive penetrability of posture control. Experimental Aging Research, 19(1), 1-13.

Teasdale, N., Stelmach, G. E., Bard, C., \& Fleury, M. (1992). Posture and elderly persons: Deficits in the central integrative mechanisms. In $\mathrm{M}$. Woollacott, \& F. Horak (Eds.), Posture and gait: Control mechanisms (vol. II, pp. 203-207). Portland, Oregon: University of Oregon Books.

Tell, G. S., Lefkowitz, D. S., Diehr, P., \& Elster, A. D. (1998). Relationship between balance and abnormalities in cerebral magnetic resonance imaging in older adults. Archives of Neurology, 55, 73-79.

Van Wegen, E. E. H., Emmerik, R. E. A., \& Riccio, G. E. (2002). Postural orientation: Age-related changes in variability and time-to-boundary. Human Movement Science, 21, 61-84.

Voelcker-Rehage, C., \& Alberts, J. L. (2007). Effect of motor practice on dual-task performance in older adults. Journal of Gerontology: Psychological Sciences, 62(3), 141-148.

Woollacott, M. H., \& Shumway-Cook, A. (2002). Attention and the control of posture and gait: A review of an emerging area of research. Gait \& Posture, 16, 1-14.

(c) EY-No Todo o conteúdo da revista Motricidade está licenciado sob a Creative Commons, excepto quando especificado em contrário e nos conteúdos retirados de outras fontes bibliográficas. 